VOL. 55 (1997) [299-309]

\title{
ON SOME MAPPINGS ASSOCIATED WITH GEOMETRIC AND ARITHMETIC MEANS
}

\author{
S.S. Dragomir, D. Comànescu and C.E.M. Pearce
}

In this paper we introduce some new mappings associated with the weighted geometric mean. These are used to derive structural results linking weighted geometric and arithmetic means.

\section{INTRODUCTION}

Considerable attention has been given in the literature to studying relations between various means. Thus if $G(x, y), A(x, y), L(x, y)$ denote respectively the geometric, arithmetic and logarithmic means of two positive numbers $x, y$, the relation

$$
\left\{[G(x, y)]^{2} A(x, y)\right\}^{1 / 3} \leqslant L(x, y)
$$

applies (see Leach and Sholander [4]). A variety of recent results has been assembled by Mitrinović, Pečarić and Fink [6, Chapter 2].

The fundamental inequality in the area remains the familiar arithmetico-geometric inequality

$$
A_{n}(p, x) \geqslant G_{n}(p, x), \quad x \in \mathbb{R}_{+}^{n} .
$$

Here $x=\left(x_{1}, \ldots, x_{n}\right) \in \mathbb{R}_{+}^{n}$ and $p \in \mathbb{R}_{+}^{n}$, with $P_{n}:=\sum_{i=1}^{n} p_{i}>0$ and

$$
A_{n}(p, x):=\frac{1}{P_{n}} \sum_{i=1}^{n} p_{i} x_{i} \text { and } G_{n}(p, x):=\left(\prod_{i=1}^{n} x_{i}^{p_{i}}\right)^{1 / P_{n}}
$$

are respectively the arithmetic and geometric means of the finite sequence $x$ with the weights $p$.

As an indication of its central role, Bullen, Mitrinović and Vasić devote most of a chapter of their book [1] to this result, including treatments of some 50-odd known proofs. The reason for this largesse is that different methods suggest different extensions and improvements. (In this connection, we shall, perhaps, be forgiven for opening

Received 18th April, 1996

Copyright Clearance Centre, Inc. Serial-fee code: 0004-9729/97 \$A2.00+0.00. 
Section 2 with a particularly simple proof of a result which, while new, is subsumed under a later and more general one established by a different method.) Extensions of (1.1) include bounds on $A_{n} / G_{n}$ and $A_{n}-G_{n}$ (see [1, Chapter 2]). For example, if $K$ is the ratio of the greatest to the least of the values $x_{i}$, then for the case of equal weights $p_{i}$ we have

$$
1 \leqslant \frac{A_{n}(p, x)}{G_{n}(p, x)} \leqslant \frac{(K-1) K^{1 /(K-1)}}{e \ln K}
$$

(see Dočev [2]).

This article is in the spirit of the above developments, though we find that $A_{n} / G_{n}$ and $A_{n}-G_{n}$ are not the most natural quantities with which to work. Also, in contrast to most of the work referred to, which studies relations between means of fixed real positive numbers, we shall address results relating to means considered as functions of variables. Thus it is obvious that

$$
A_{n}(p, x+y)=A_{n}(p, x)+A_{n}(p, y) \text { and } A_{n}(p, \alpha x)=\alpha A_{n}(p, x)
$$

for all $p, x, y \in \mathbb{R}_{+}^{n}$ with $P_{n}>0$ and $\alpha>0$, that is, the mapping $A_{n}(p, \cdot)$ is additive and positive homogeneous on $\mathbb{R}_{+}^{n}$. Furthermore

$$
G_{n}(p, x \cdot y)=G_{n}(p, x) \cdot G_{n}(p, y) \text { and } G_{n}(p, \alpha x)=\alpha G_{n}(p, x)
$$

for all $p, x, y \in \mathbb{R}_{+}^{n}$ with $P_{n}>0$ and $\alpha>0$, that is, the mapping $G_{n}(p, \cdot)$ is multiplicative and positive homogeneous on $\mathbb{R}_{+}^{n}$.

The mapping $G_{n}(p, \cdot)$ possesses also a superadditivity property which may be used to uncover a richer variety of structural results. In the following section, we derive a superadditivity property for geometric means and use this to develop a supermultiplicativity property for the map $\eta_{n}(p, \cdot)$ given by

$$
\eta_{n}(p, x):=\left(\frac{G_{n}(p, x)}{A_{n}(p, x)}\right)^{A_{n}(p, x)}
$$

and a subadditivity property for the map $\mu_{n}(p, \cdot)$ given by

$$
\mu_{n}(p, x):=\frac{\left[A_{n}(p, x)\right]^{2}}{G_{n}(p, x)} .
$$

In Section 3 we adopt a motif which has proved useful in the literature for interpolation, namely, the introduction of a real variable as a parameter. This is used for the further development of the ideas of Section 2. 


\section{QUASI-ADDITIVITY AND QUASI-MULTIPLICATIVITY PROPERTIES}

First we consider the so-called unweighted (that is, uniformly weighted) geometric mean

$$
G_{n}(x):=\prod_{i=1}^{n} x_{i}^{1 / n}
$$

The following theorem expresses superadditivity and monotonicity properties of the geometric mean $G_{n}$.

TheOREM 2.1. Let $n \geqslant 2$ be a natural number. Then the mapping $G_{n}(\cdot)$ is superadditive and monotone nondecreasing on $\mathbb{R}_{+}^{n}$.

ProOF: If there exists $i_{0} \in\{1, \ldots, n\}$ with $y_{i_{0}}=0$, then the inequality

$$
G_{n}(x+y) \geqslant G_{n}(x)+G_{n}(y)
$$

holds trivially. On the other hand, suppose that $y_{i}>0$ for all $i \in\{1, \ldots, n\}$. If $a$ is a nonnegative $n$-vector and $1+a:=\left(1+a_{1}, \ldots, 1+a_{n}\right)$, then

$$
1+G_{n}(a) \leqslant G_{n}(1+a)
$$

(Lupaş and Mitrović [5] and Kečkić [3]). For a more accessible account see Bullen, Mitrinović and Vasić [1, Chapter 2, Theorem 19].

Put $a_{i}=x_{i} / y_{i}(1 \leqslant i \leqslant n)$. It follows that

$$
\frac{G_{n}(x)+G_{n}(y)}{G_{n}(y)}=1+\prod_{i=1}^{n}\left(\frac{x_{i}}{y_{i}}\right)^{1 / n} \leqslant \prod_{i=1}^{n}\left(1+\frac{x_{i}}{y_{i}}\right)^{1 / n}=\frac{G_{n}(x+y)}{G_{n}(y)}
$$

and again (2.1) holds, establishing superadditivity.

We deduce in particular that if $x \geqslant y$ then

$$
G_{n}(x) \geqslant G_{n}(x-y)+G_{n}(y)
$$

and so, as $G_{n}(x-y) \geqslant 0$, that

$$
G_{n}(x) \geqslant G_{n}(y)
$$

which establishes monotonicity.

In fact equality holds in (2.2) only if $a_{1}=\ldots=a_{n}$, so that if $x$ and $y$ are strictly positive vectors then strict inequality holds in (2.1) and (2.3) unless $x_{i}=y_{i}$ $(1 \leqslant i \leqslant n)$.

We now show that these results hold when $G_{n}(\cdot)$ is replaced by $G_{n}(p, \cdot)$. 
THEOREM 2.2. Let $n \geqslant 2$ be a natural number and $p \in \mathbb{R}_{+}^{n}$ with $P_{n}>0$. Then the mapping $G_{n}(p, \cdot)$ is superadditive and monotone nondecreasing on $\mathbb{R}_{+}^{n}$.

Proof: As in the previous theorem, it is enough to treat the case in which $x_{i}, y_{i}$ are strictly positive for all $i \in\{1, \ldots, n\}$. Consider the mapping $f: \mathbb{R} \rightarrow(0, \infty)$ given by $f(x)=\ln \left(1+e^{x}\right)$. We have

$$
f^{\prime}(x)=\frac{e^{x}}{1+e^{x}} \text { and } f^{\prime \prime}(x)=\frac{e^{x}}{\left(1+e^{x}\right)^{2}} \text { for all } x \in \mathbb{R},
$$

which shows that the mapping $f$ is convex on $\mathbb{R}$.

Jensen's inequality

$$
\frac{1}{P_{n}} \sum_{i=1}^{n} p_{i} f\left(a_{i}\right) \geqslant f\left(\frac{1}{P_{n}} \sum_{i=1}^{n} p_{i} a_{i}\right)
$$

provides

$$
\left[\prod_{i=1}^{n}\left(1+\exp \left(a_{i}\right)\right)^{p_{i}}\right]^{1 / P_{n}} \geqslant 1+\exp \left(\frac{1}{P_{n}} \sum_{i=1}^{n} p_{i} a_{i}\right) .
$$

With the choice $a_{i}=\ln \left(x_{i} / y_{i}\right), 1 \leqslant i \leqslant n$, this yields

$$
\left[\prod_{i=1}^{n}\left(1+\frac{x_{i}}{y_{i}}\right)^{p_{i}}\right]^{1 / P_{n}} \geqslant 1+\left[\prod_{i=1}^{n}\left(\frac{x_{i}}{y_{i}}\right)^{p_{i}}\right]^{1 / P_{n}}
$$

which gives us

$$
\frac{G_{n}(p, x+y)}{G_{n}(p, y)} \geqslant \frac{G_{n}(p, x)+G_{n}(p, y)}{G_{n}(p, y)} .
$$

The remainder of the proof proceeds as before.

By way of corollary, the standard condition for equality to apply in Jensen's inequality again tells us that if $x$ and $y$ are strictly positive vectors, then strict inequality holds in both (2.1) and (2.3) (for $x \geqslant y$ ) unless $x_{i}=y_{i}(1 \leqslant i \leqslant n)$.

We shall now use the superadditivity of geometric means together with the additivity of arithmetic means to derive a further result. We define the mapping

$$
\eta_{n}(p, x):=\left(\frac{G_{n}(p, x)}{A_{n}(p, x)}\right)^{A_{n}(p, x)} \text { for } p, x \geqslant 0 \text { and } A_{n}(p, x)>0 .
$$

Here and subsequently it will be helpful to invoke the auxiliary quantity

$$
L_{n}(p, x):=\frac{G_{n}(p, x)}{A_{n}(p, x)} .
$$


ThEOREM 2.3. Let $p \in \mathbb{R}_{+}^{n}$ with $P_{n}>0$ and $n \geqslant 2$. Then $\eta_{n}(p, \cdot)$ is supermultiplicative on $\mathbb{R}_{+}^{n}$.

Proof: By the superadditivity of the geometric mean and the additivity of the arithmetic mean, we have for $A_{n}(p, x)>0, A_{n}(p, y)>0$ that

$$
\begin{aligned}
L_{n}(p, x+y) & \geqslant \frac{G_{n}(p, x)+G_{n}(p, y)}{A_{n}(p, x)+A_{n}(p, y)} \\
& =\frac{A_{n}(p, x) L_{n}(p, x)+A_{n}(p, y) L_{n}(p, y)}{A_{n}(p, x)+A_{n}(p, y)} \\
& \geqslant\left[L_{n}(p, x)\right]^{A_{n}(p, x) / A_{n}(p, x+y)} \cdot\left[L_{n}(p, y)\right]^{A_{n}(p, y) / A_{n}(p, x+y)} .
\end{aligned}
$$

Thus

$$
\left[L_{n}(p, x+y)\right]^{A_{n}(p, x+y)} \geqslant\left[L_{n}(p, x)\right]^{A_{n}(p, x)}\left[L_{n}(p, y)\right]^{A_{n}(p, y)}
$$

or

$$
\eta_{n}(p, x+y) \geqslant \eta_{n}(p, x) \cdot \eta_{n}(p, y)
$$

which establishes the desired result.

REMARK 2.4. Define the mapping $\bar{\eta}_{n}(p, \cdot)$ by $\bar{\eta}_{n}(p, x)=\left[\eta_{n}(p, x)\right]^{-1}$. Then $\bar{\eta}_{n}(p, x) \geqslant$ 1 and $\bar{\eta}_{n}(p, \cdot)$ is submultiplicative.

We can introduce also a mapping $\mu_{n}(p, \cdot)$ by

$$
\mu_{n}(p, x):=\frac{\left[A_{n}(p, x)\right]^{2}}{G_{n}(p, x)}
$$

where $P_{n} \geqslant 0$ and $x_{i}>0$ for $1 \leqslant i \leqslant n$.

THEOREM 2.4. Let $p \in \mathbb{R}_{+}^{n}$ with $P_{n}>0$ and $n \geqslant 2$. Then the mapping $\mu_{n}(p, \cdot)$ is subadditive and positive homogeneous.

Proof: Let $x, y \in \mathbb{R}_{+}^{n}$ with $x_{i}, y_{i} \neq 0$ for all $i \in\{1, \ldots, n\}$. We have, by the superadditivity of the mapping $G_{n}(p, \cdot)$ and the additivity of $A_{n}(p, \cdot)$, that

$$
\begin{array}{r}
\mu(p, x+y)=\frac{\left[A_{n}(p, x+y)\right]^{2}}{G_{n}(p, x+y)} \leqslant \frac{\left(A_{n}(p, x)+A_{n}(p, y)\right)^{2}}{G_{n}(p, x)+G_{n}(p, y)} \\
=\frac{\left(A_{n}(p, x)+A_{n}(p, y)\right)^{2}}{A_{n}(p, x) L_{n}(p, x)+A_{N}(p, y) L_{n}(p, y)} .
\end{array}
$$

We now apply the arithmetico-harmonic mean inequality

$$
\frac{\alpha a+\beta b}{\alpha+\beta} \geqslant \frac{\alpha+\beta}{\alpha / a+\beta / b}, \quad a, b>0 \text { and } \alpha, \beta \geqslant 0 \text { with } \alpha+\beta>0,
$$


which it is convenient to recast as

$$
\frac{(\alpha+\beta)^{2}}{\alpha / a+\beta / b} \leqslant \alpha a+\beta b
$$

For $\alpha=A_{n}(p, x), \beta=A_{n}(p, y), a=1 / L_{n}(p, x)$ and $b=1 / L_{n}(p, y)$ this gives

$$
\frac{\left(A_{n}(p, x)+A_{n}(p, y)\right)^{2}}{A_{n}(p, x) L_{n}(p, x)+A_{n}(p, y) L_{n}(p, y)} \leqslant \frac{A_{n}(p, x)}{L_{n}(p, x)}+\frac{A_{n}(p, y)}{L_{n}(p, y)}
$$

that is,

$$
\mu_{n}(p, x+y) \leqslant \mu_{n}(p, x)+\mu_{n}(p, y),
$$

providing subadditivity. The condition $\mu_{n}(p, \alpha x)=\alpha \mu_{n}(p, x)$ for positive homogeneity is immediate.

\section{Some MAPPINGS OF A REAL VARIABLE}

In this section we introduce a real variable as a parameter to continue the ideas of the previous two theorems. First we define a mapping $\eta$ of a real variable by

$$
\eta(t):=\left[\frac{G_{n}(p, x+t e) A_{n}(p, e)}{A_{n}(p, x+t e) G_{n}(p, e)}\right]^{A_{n}(p, x+t e)}=\left[\frac{L_{n}(p, x+t e)}{L_{n}(p, e)}\right]^{A_{n}(p, x+t e)}
$$

for $e$ a fixed positive $n$-vector and $t \geqslant 0$.

The following theorem encapsulates its main properties.

THEOREM 3.1. The mapping $\eta$ is monotonic nondecreasing and logarithmically concave. Further, it satisfies the estimate

$$
\left[\frac{L_{n}(p, x)}{L_{n}(p, e)}\right]^{A_{n}(p, x)} \leqslant \eta(t) \leqslant \exp \left[A_{n}(p, e) A_{n}\left(p, \frac{x}{e}\right)-A_{n}(p, x)\right]
$$

for all $t \in[0, \infty)$, where as in Bullen, Mitrinović and Fink [1] we define $x / e:=$ $\left(x_{1} / e_{1}, \ldots, x_{n} / e_{n}\right)$.

Proof: Suppose that $t_{2}>t_{1} \geqslant 0$. The positive homogeneity of $A_{n}(p, \cdot)$ and $G_{n}(p, \cdot)$ induces the affine property

$$
L_{n}(p, \alpha v)=L_{n}(p, v)
$$

for $\alpha>0$, so that

$$
L_{n}\left(p,\left(t_{2}-t_{1}\right) e\right)=L_{n}(p, e) \text { for } t_{2}>t_{1}
$$


Hence

$$
\begin{aligned}
& \eta\left(t_{2}\right) \\
& =\eta\left(t_{1}+\left(t_{2}-t_{1}\right)\right) \\
& =L_{n}\left(p, x+t_{1} e+\left(t_{2}-t_{1}\right) e\right)^{A_{n}\left(p, x+t_{1} e\right)+\left(t_{2}-t_{1}\right) A_{n}(x, e)} L_{n}(p, e)^{-A_{n}\left(p, x+t_{1} e\right)-\left(t_{2}-t_{1}\right) A_{n}(x, e)} \\
& \geqslant \\
& \quad L_{n}\left(p, x+t_{1} e\right)^{A_{n}\left(p, x+t_{1} e\right)} L_{n}\left(p,\left(t_{2}-t_{1}\right) e\right)^{\left(t_{2}-t_{1}\right) A_{n}(x, e)} L_{n}(p, e)^{-A\left(p, x+t_{1} e\right)} \\
& \quad \times L_{n}(p, e)^{-\left(t_{2}-t_{1}\right) A_{n}(x, e)} \\
& =\eta\left(t_{1}\right)
\end{aligned}
$$

and the monotonicity of $\eta$ is thus proved.

Now let $\alpha, \beta \geqslant 0$ with $\alpha+\beta=1$ and $t_{1}, t_{2} \in[0, \infty)$. Then we have similarly

$$
\begin{aligned}
\eta\left(\alpha t_{1}+\beta t_{2}\right)= & L_{n}\left(p, \alpha\left(x+t_{1} e\right)+\beta\left(x+t_{2} e\right)\right)^{A_{n}\left(p, \alpha\left(x+t_{1} e\right)+\beta\left(x+t_{2} e\right)\right)} \\
& \times L_{n}(p, e)^{-\alpha A_{n}\left(p, x+t_{1} e\right)-\beta A_{n}\left(p, x+t_{2} e\right)} \\
\geqslant & L_{n}\left(p, \alpha\left(x+t_{1} e\right)\right)^{A_{n}\left(p, \alpha\left(x+t_{1} e\right)\right)} L_{n}\left(p, \beta\left(x+t_{2} e\right)\right)^{A_{n}\left(p, \beta\left(x+t_{2} e\right)\right)} \\
& \times L_{n}(p, e)^{-\alpha A_{n}\left(p, x+t_{1}\right)} L_{n}(p, e)^{-\beta A_{n}\left(p, x+t_{2} e\right)} \\
= & \left\{\left[\frac{L_{n}\left(p, x+t_{1} e\right)}{L_{n}(p, e)}\right]^{A_{n}\left(p, x+t_{1} e\right)}\right\}^{\alpha} \times\left\{\left[\frac{L_{n}\left(p, x+t_{2} e\right)}{L_{n}(p, e)}\right]^{A_{n}\left(p, x+t_{2} e\right)}\right\}^{\beta} \\
= & {\left[\eta\left(t_{1}\right)\right]^{\alpha}\left[\eta\left(t_{2}\right)\right]^{\beta} . }
\end{aligned}
$$

Thus the mapping $\eta$ is logarithmically concave.

The first inequality in (3.1) follows from the fact that $\eta$ is monotonic nondecreasing on $[0, \infty)$ and $\eta(0)=\left[L_{n}(p, x) / L_{n}(p, e)\right]^{A_{n}(p, x)}$.

To prove the second, define the mapping

$$
m(t):=\frac{L_{n}(p, x+t e)}{L_{n}(p, e)}-1, \quad t \geqslant 0 .
$$

We have easily that

$$
\lim _{t \rightarrow \infty} \frac{G_{n}(p, x+t e)}{t}=\lim _{t \rightarrow \infty}\left[\prod_{i=1}^{n}\left(\frac{x_{i}+t e_{i}}{t}\right)^{p_{i}}\right]^{1 / P_{n}}=G_{n}(p, e)
$$

and

$$
\lim _{t \rightarrow \infty} \frac{A_{n}(p, x+t e)}{t}=A_{n}(p, e) .
$$

Thus $L_{n}(p, x+t e) \rightarrow L_{n}(p, e)$ as $t \rightarrow \infty$ and so $\lim _{t \rightarrow \infty} m(t)=0$. Consequently we have that

$$
\lim _{t \rightarrow \infty} \eta(t)=\lim _{t \rightarrow \infty}\left\{(1+m(t))^{1 / m(t)}\right\}^{m(t) A_{n}(p, x+t e)}=\exp \left[\lim _{t \rightarrow \infty}\left(m(t) A_{n}(p, x+t e)\right)\right] .
$$


Further,

$$
\begin{aligned}
\lim _{t \rightarrow \infty}\left[m(t) A_{n}(p, x+t e)\right] & =\lim _{t \rightarrow \infty}\left[\frac{G_{n}(p, x+t e) A_{n}(p, e)}{G_{n}(p, e)}-A_{n}(p, x+t e)\right] \\
& =\lim _{u \rightarrow 0} \frac{G_{n}(p, u x+e) A_{n}(p, e)-A_{n}(p, u x+e) G_{n}(p, e)}{u G_{n}(p, e)} .
\end{aligned}
$$

Consider the mapping

$h(u):=G_{n}(p, u x+e)=\left[\prod_{i=1}^{n}\left(u x_{i}+e_{i}\right)^{p_{i}}\right]^{1 / P_{n}}=\exp \left[\frac{1}{P_{n}} \sum_{i=1}^{n} p_{i} \ln \left(u x_{i}+e_{i}\right)\right], \quad u \geqslant 0$.

We have for $u \geqslant 0$ that

$$
\begin{aligned}
\frac{d h(u)}{d u} & =\left[\exp \left(\frac{1}{P_{n}} \sum_{i=1}^{n} p_{i} \ln \left(u x_{i}+e_{i}\right)\right)\right] \frac{1}{P_{n}} \sum_{i=1}^{n} p_{i} \frac{x_{i}}{u x_{i}+e_{i}} \\
& =G_{n}(p, u x+e) \cdot A_{n}\left(p, \frac{x}{u x+e}\right) .
\end{aligned}
$$

Using L'Hôpital's rule, we get

$$
\begin{aligned}
\lim _{u \rightarrow 0+} \frac{G_{n}(p, u x+e) A_{n}(p, e)-A_{n}(p, u x+e) G_{n}(p, e)}{u G_{n}(p, e)} \\
\quad=\lim _{u \rightarrow 0+} \frac{A_{n}(p, e) \frac{d}{d u}\left(G_{n}(p, u x+e)\right)-G_{n}(p, e) \frac{d}{d u}\left(A_{n}(p, u x+e)\right)}{G_{n}(p, e)} \\
=\frac{G_{n}(p, e) A_{n}(p, e) A_{n}\left(p, \frac{x}{e}\right)-G_{n}(p, e) A_{n}(p, x)}{G_{n}(p, e)} \\
=A(p, e) A_{n}\left(p, \frac{x}{e}\right)-A_{n}(p, x) .
\end{aligned}
$$

Finally, we get the limit

$$
\lim _{t \rightarrow \infty} \eta(t)=\exp \left[A_{n}(p, e) A_{n}\left(p, \frac{x}{e}\right)-A_{n}(p, x)\right] .
$$

Since $\eta$ is monotonic nondecreasing, we have the desired inequality and the proof is finished.

Now, we introduce another mapping of a real variable

$$
\mu(t):=\frac{\left[A_{n}(p, x+t e)\right]^{2} G_{n}(p, e)}{G_{n}(p, x+t e)\left[A_{n}(p, e)\right]^{2}}=\frac{\mu_{n}(p, x+e t)}{\mu_{n}(p, e)}, t \geqslant 0,
$$

where $p, x, e$ are as above.

The main properties of this mapping are embodied in the following theorem. 


\section{THEOREM 3.2. With the above assumptions}

(i) the mapping $\mu$ is convex on $[0, \infty)$;

(ii) the mapping $\mu-\mathbb{1}$ is monotonic nonincreasing on $[0, \infty)$, where $\mathbb{1}(t)=t$, $t \in \mathbb{R}$;

(iii) we have the estimate

$$
\frac{\left[A_{n}(p, x)\right]^{2} G_{n}(p, e)}{G_{n}(p, x)\left[A_{n}(p, e)\right]^{2}}+t \geqslant \mu(t)>t+\frac{2 A_{n}(p, x)-A_{n}\left(p, \frac{x}{e}\right) A_{n}(p, e)}{A_{n}(p, e)}
$$

for all $t \in[0, \infty)$.

Proof: (i) Let $\alpha, \beta \geqslant 0$ with $\alpha+\beta=1$ and $t_{1}, t_{2} \in[0, \infty)$. Then we have

$$
\begin{aligned}
\mu\left(\alpha t_{1}+\beta t_{2}\right) & =\frac{\mu_{n}\left(p, \alpha\left(x+t_{1} e\right)+\beta\left(x+t_{2} e\right)\right)}{\mu_{n}(p, e)} \\
& \leqslant \frac{\alpha \mu_{n}\left(p, x+t_{1} e\right)+\beta \mu_{n}\left(p, x+t_{2} e\right)}{\mu_{n}(p, e)} \\
& =\alpha \mu\left(t_{1}\right)+\beta \mu\left(t_{2}\right)
\end{aligned}
$$

which proves that $\mu$ is convex on $[0, \infty)$.

(ii) Now let $t_{2}>t_{1} \geqslant 0$. Then

$$
\begin{aligned}
\mu\left(t_{2}\right) & =\mu\left(t_{1}+\left(t_{2}-t_{1}\right)\right) \\
& =\frac{\mu_{n}\left(p, x+t_{1} e+\left(t_{2}-t_{1}\right) e\right)}{\mu_{n}(p, e)} \\
& \leqslant \frac{\mu_{n}\left(p, x+t_{1} e\right)+\left(t_{2}-t_{1}\right) \mu_{n}(p, x)}{\mu_{n}(p, x)} \\
& =\mu\left(t_{1}\right)+t_{2}-t_{1},
\end{aligned}
$$

which gives us

$$
\mu\left(t_{2}\right)-t_{2} \leqslant \mu\left(t_{1}\right)-t_{1} \text { for all } t_{2}>t_{1} \geqslant 0
$$

and the monotonicity of the mapping $\mu-\mathbb{1}$ is proved.

(iii) The first inequality in (3.2) follows to the fact that $\mu-\mathbb{1}$ is monotonic nonincreasing and

$$
\mu(0)=\frac{\left[A_{n}(p, x)\right]^{2} G_{n}(p, e)}{G_{n}(p, x)\left[A_{n}(p, e)\right]^{2}} .
$$

For the second, we proceed as follows. 
For all $t>0$ we have

$$
\begin{aligned}
\mu(t)-t & =\frac{\left[A_{n}(p, x+t e)\right]^{2} G_{n}(p, e)-t G_{n}(p, x+t e)\left[A_{n}(p, e)\right]^{2}}{G_{n}(p, x+t e)\left[A_{n}(p, e)\right]^{2}} \\
& =\frac{\frac{1}{t}\left[A_{n}\left(p_{1} x+t e\right)\right]^{2} G_{n}(p, e)-G_{n}(p, x+t e)\left[A_{n}(p, t)\right]^{2}}{\frac{G_{n}(p, x+t e)}{t}\left[A_{n}(p, e)\right]^{2}}
\end{aligned}
$$

As in Theorem 3.1, we have

$$
\lim _{t \rightarrow \infty} \frac{G_{n}(p, x+t e)}{t}=G_{n}(p, e)
$$

Let us compute the limit

$$
I=\lim _{t \rightarrow \infty}\left\{\frac{1}{t}\left[A_{n}(p, x+t e)\right]^{2} G_{n}(p, e)-G_{n}(p, x+t e)\left[A_{n}(p, e)\right]^{2}\right\} .
$$

We have

$$
\begin{aligned}
I & =\lim _{u \rightarrow 0+}\left\{u\left[A_{n}\left(p, x+\frac{1}{u} e\right)\right]^{2} G_{n}(p, e)-G_{n}\left(p, x+\frac{1}{u} e\right)\left[A_{n}(p, e)\right]^{2}\right\} \\
& =\lim _{u \rightarrow 0+}\left\{\frac{1}{u}\left[A_{n}(p, u x+e)\right]^{2} G_{n}(p, e)-\frac{1}{u} G_{n}(p, u x+e)\left[A_{n}(p, e)\right]^{2}\right\} \\
& =\lim _{u \rightarrow 0+} \frac{\left[A_{n}(p, u x+e)\right]^{2} G_{n}(p, e)-G_{n}(p, u x+e)\left[A_{n}(p, e)\right]^{2}}{u} .
\end{aligned}
$$

Also we have

$$
\frac{d}{d u}\left[A_{n}(p, u x+e)\right]^{2}=2 A_{n}(p, u x+e) A_{n}(p, x)
$$

and as in Theorem 3.1

$$
\frac{d}{d u}\left[G_{n}(p, u x+e)\right]=G_{n}(p, u x+e) A_{n}\left(p, \frac{x}{u x+e}\right) .
$$

Applying L'Hôpital's rule we get

$$
\begin{aligned}
I & =\lim _{u \rightarrow 0+}\left[2 A_{n}(p, u x+e) A_{n}(p, x) G_{n}(p, e)-G_{n}(p, u x+e) A_{n}\left(p, \frac{x}{u x+e}\right)\left[A_{n}(p, e)\right]^{2}\right] \\
& =2 A_{n}(p, e) A_{n}(p, x) G_{n}(p, e)-G_{n}(p, e) A_{n}\left(p, \frac{x}{e}\right)\left[A_{n}(p, e)\right]^{2} \\
& =A_{n}(p, e) G_{n}(p, e)\left[2 A_{n}(p, x)-A_{n}\left(p, \frac{x}{e}\right) A_{n}(p, e)\right]
\end{aligned}
$$

and thus

$$
\begin{aligned}
\lim _{t \rightarrow \infty}[\mu(t)-t] & =\frac{A_{n}(p, e) G_{n}(p, e)\left[2 A_{n}(p, x)-A_{n}\left(p, \frac{x}{e}\right) A_{n}(p, e)\right]}{G_{n}(p, e)\left[A_{n}(p, e)\right]^{2}} \\
& =\frac{2 A_{n}(p, x)-A_{n}\left(p, \frac{x}{e}\right) A_{n}(p, e)}{A_{n}(p, e)} .
\end{aligned}
$$

As the mapping $\mu-\mathbb{1}$ is monotonic nondecreasing we get the second part of (3.2) and the proof is finished. 
[11] Mappings associated with geometric and arithmetic means

\section{REFERENCES}

[1] P.S. Bullen, D.S. Mitrinović and P.M. Vasić, Means and their inequalities (Kluwer Academic Publishers, Dordrecht, 1988).

[2] K. Dočev, 'Problem 95', Fiz.-Mat. Spis. Bulgar. Akad. Nauk. 9 (1966), 54-55.

[3] J.D. Kečkić, 'A method of forming inequalities', Math. Balkanica 1 (1971), 140-141.

[4] E.B. Leach and M.C. Sholander, 'Extended mean values II', J. Math. Anal. Appl. 92 (1983), 207-223.

[5] A. Lupaş and Z. Mitrović, 'Problem 156', Mat. Vesnik 21 (1969), p. 242.

[6] D.S. Mitrinovič, J.E. Pečarić and A.M. Fink, Classical and new inequalities in analysis (Kluwer Academic Publishers, Dordrecht, 1993).

Faculty of Mathematics

University of Timişoara

RO-1900, Timişoara

Romania

Department of Applied Mathematics

The University of Adelaide

South Australia 5005

Australia
Faculty of Mathematics

University of Timişoara

RO-1900, Timişoara

Romania 